INFECTION CONTROL \& HOSPITAL EPIDEMIOLOGY NOVEMBER 2016, Vol. 37, No. 11

\title{
Antimicrobial-Resistant Pathogens Associated With Healthcare-Associated Infections: Summary of Data Reported to the National Healthcare Safety Network at the Centers for Disease Control and Prevention, 2011-2014
}

\author{
Lindsey M. Weiner, MPH; Amy K. Webb, MPH, CHES; Brandi Limbago, PhD; Margaret A. Dudeck, MPH, CPH; \\ Jean Patel, PhD; Alexander J. Kallen, MD, MPH; Jonathan R. Edwards, MStat; Dawn M. Sievert, PhD, MS
}

овјестіле. To describe antimicrobial resistance patterns for healthcare-associated infections (HAIs) that occurred in 2011-2014 and were reported to the Centers for Disease Control and Prevention's National Healthcare Safety Network.

methods. Data from central line-associated bloodstream infections, catheter-associated urinary tract infections, ventilator-associated pneumonias, and surgical site infections were analyzed. These HAIs were reported from acute care hospitals, long-term acute care hospitals, and inpatient rehabilitation facilities. Pooled mean proportions of pathogens that tested resistant (or nonsusceptible) to selected antimicrobials were calculated by year and HAI type.

RESUlts. Overall, 4,515 hospitals reported that at least 1 HAI occurred in 2011-2014. There were 408,151 pathogens from 365,490 HAIs reported to the National Healthcare Safety Network, most of which were reported from acute care hospitals with greater than 200 beds. Fifteen pathogen groups accounted for $87 \%$ of reported pathogens; the most common included Escherichia coli (15\%), Staphylococcus aureus (12\%), Klebsiella species (8\%), and coagulase-negative staphylococci (8\%). In general, the proportion of isolates with common resistance phenotypes was higher among device-associated HAIs compared with surgical site infections. Although the percent resistance for most phenotypes was similar to earlier reports, an increase in the magnitude of the resistance percentages among E. coli pathogens was noted, especially related to fluoroquinolone resistance.

CONCLUSION. This report represents a national summary of antimicrobial resistance among select HAIs and phenotypes. The distribution of frequent pathogens and some resistance patterns appear to have changed from 2009-2010, highlighting the need for continual, careful monitoring of these data across the spectrum of HAI types.

Infect Control Hosp Epidemiol 2016;37:1288-1301

In 2005, the Centers for Disease Control and Prevention (CDC) launched the National Healthcare Safety Network (NHSN), a system used by the CDC, healthcare facilities, state health departments, the Centers for Medicare and Medicaid Services (CMS), and other organizations for surveillance of patient and healthcare personnel safety. NHSN's surveillance coverage includes a variety of healthcare-associated infections (HAIs), each of which can be reported by acute care hospitals and other healthcare facilities. In its 10 years of operational use, NHSN has grown to become the single largest HAI surveillance system in the United States, with more than 17,000 healthcare facilities of varying types participating and all 50 states represented.

Antimicrobial susceptibility test results for pathogens implicated in HAIs are an important source of information about the scope and magnitude of emerging and endemic antimicrobial-resistant infections in the United States. Analysis of NHSN data provides summary measures of antimicrobial resistance prevalence; these can help inform decisions about infection prevention practice, antimicrobial development and stewardship, and public policies aimed at detecting and preventing transmission of resistant strains and/or their resistance determinants, especially those with phenotypes having the fewest viable treatment options.

This report is the third summary of NHSN antimicrobial susceptibility data and is based on data reported to NHSN for HAIs that occurred in 2011-2014. This period coincides with an increased use of NHSN by acute care hospitals, long-term acute care hospitals (LTACHs), and inpatient rehabilitation facilities (IRFs) due to new HAI reporting requirements for participation in CMS Quality Reporting Programs (QRPs).

Affiliation: Division of Healthcare Quality Promotion, National Center for Emerging and Zoonotic Infectious Diseases, Centers for Disease Control and Prevention, Atlanta, Georgia. (Present affiliation: Lantana Consulting Group, East Thetford, Vermont [D.M.S.].)

Received June 8, 2016; accepted June 17, 2016; electronically published August 30, 2016

(c) 2016 by The Society for Healthcare Epidemiology of America. All rights reserved. 0899-823X/2016/3711-0004. DOI: 10.1017/ice.2016.174 
This report follows the basic methodology of the first 2 reports $^{1,2}$ and provides additional and updated susceptibility results for select pathogens reported to NHSN. This report complements other NHSN summary reports including the annual summary of infection rates from the Device-Associated Module, ${ }^{3}$ and the national and state-specific HAI progress reports. ${ }^{4}$ In addition, the types of antimicrobial resistance data included in this report are used to inform national estimates such as those published in CDC's Antibiotic Threat Report, ${ }^{5}$ which presents antimicrobial resistance data from multiple surveillance sources in a comprehensive overview of resistant infections in the United States. Some of these data are also incorporated into the Antibiotic Resistance Patient Safety Atlas, which allows for a detailed review of specific resistance data (available at http://www.cdc.gov/hai/surveillance/ ar-patient-safety-atlas.html).

\section{METHODS}

HAIs that occurred in 2011-2014 and were reported to the Device-Associated and Procedure-Associated Modules of the Patient Safety Component of $\mathrm{NHSN}^{6-9}$ as of December 16, 2015, were included in this report. These HAIs were reported from acute care hospitals, LTACHs, and IRFs, and include central line-associated bloodstream infections (CLABSIs), catheterassociated urinary tract infections (CAUTIs), all surgical site infections (SSIs) following inpatient procedures with a primary closure technique, and ventilator-associated pneumonias (VAPs). VAP surveillance in adult locations was retired from NHSN in January 2013 and was replaced with the surveillance of ventilator-associated events (VAEs). Therefore, VAP data in this report are limited to events in 2011-2012, and this will be the last report to include such data. Postprocedure pneumonias, asymptomatic bacteremic urinary tract infections, and pediatric VAPs, each of which accounted for less than $1 \%$ of reported HAIs, were excluded from these analyses. NHSN surveillance methodology has been reported elsewhere ${ }^{6-9}$ and is summarized in the first NHSN antimicrobial resistance report. ${ }^{1}$

Pathogen and antimicrobial susceptibility data reported to NHSN are provided by the facility's designated clinical microbiology laboratory. At most, 3 pathogens can be reported per HAI. For some pathogens, there is a select group of antimicrobials for which susceptibility test results must be reported if testing was performed. Laboratories are expected to use the current Clinical and Laboratory Standards Institute standards for antimicrobial susceptibility testing. ${ }^{10}$ Susceptibility results were reported using the category interpretations of susceptible [S], intermediate [I], resistant [R], or not tested. Because laboratories may test different antimicrobial agents within a class, for some phenotypes, resistance was defined using at least 1 of several agents within the same class.

Resistance for Staphylococcus aureus and Enterococcus spp. phenotypes included those pathogens that tested R to oxacillin, methicillin, or cefoxitin (methicillin-resistant S. aureus), or vancomycin (vancomycin-resistant Enterococcus). To be defined as resistant to extended-spectrum cephalosporins, pathogens must have tested I or R to either ceftazidime or cefepime (Pseudomonas aeruginosa) or to ceftazidime, cefepime, ceftriaxone, or cefotaxime (Enterobacteriaceae). Carbapenem resistance, as defined in this report, included all applicable pathogens with a result of I or R to imipenem, meropenem, or doripenem unless otherwise noted. Fluoroquinolone resistance was defined as a result of I or R to either ciprofloxacin or levofloxacin ( $P$. aeruginosa) or to ciprofloxacin, levofloxacin, or moxifloxacin (Escherichia coli). Aminoglycoside resistance in $P$. aeruginosa was defined as a result of I or R to gentamicin, amikacin, or tobramycin. Finally, definitions of multidrug-resistance required a test result of $\mathrm{I}$ or $\mathrm{R}$ to at least 1 agent within a class-thus establishing nonsusceptibility to the class-and nonsusceptibility to at least 3 of the specified classes. For Enterobacteriaceae species and $P$. aeruginosa, 5 classes were included in the criteria: extended-spectrum cephalosporins, fluoroquinolones, aminoglycosides, carbapenems, and piperacillin or piperacillin/tazobactam. A sixth class, ampicillin/sulbactam, was included in the criteria for multidrug-resistance for Acinetobacter spp. These criteria approximated interim standard definitions for defining multidrug-resistance. ${ }^{11}$ Results from Klebsiella pathogens were limited to K. pneumoniae and K. oxytoca combined; other species of Klebsiella were extremely rare and excluded from the analysis.

As discussed above, carbapenem-resistant Enterobacteriaceae (CRE) was defined in this report as any K. pneumoniae, K. oxytoca, E. coli, or Enterobacter spp. that tested I or R to imipenem, meropenem, or doripenem. However, this definition was updated in NHSN in 2015 to increase detection of carbapenemase-producing strains. ${ }^{12-14}$ To anticipate the impact of the updated CRE definition, the resistance percentages for CRE using both the current and updated definitions were calculated using 2014 data. In subsequent reports, CDC will use only the updated definition, which includes the above mentioned Enterobacteriaceae pathogens that test $\mathrm{R}$ to imipenem, meropenem, doripenem, or ertapenem.

Data were analyzed with SAS software, version 9.3 (SAS Institute). For reporting hospitals and all reported HAIs and pathogens, absolute frequencies and distributions were calculated by hospital characteristic, HAI, surgery, and location type. The 15 most frequently reported pathogens were identified, and their frequencies and ranks within each HAI or surgery type were calculated. For each HAI type and period, a pooled mean percent resistance was calculated for each pathogen-antimicrobial combination (ie, sum of pathogens that tested resistant, divided by the sum of pathogens tested for susceptibility, multiplied by 100). The pooled mean percent resistance was not calculated for any phenotype for which less than 20 pathogens were tested. In addition, the percentage of pathogens that were tested for susceptibility (sum of pathogens tested for susceptibility, divided by the sum of total pathogens isolated, multiplied by 100) was calculated for each pathogen-antimicrobial agent combination. 
Statistical analyses were not performed to test for temporal changes in the resistance percentage in 2011-2014, and thus, this report does not convey any definitive conclusions regarding changes in resistance over time. The results and discussions presented in this paper are based solely on observed differences in the magnitude of the resistance percentage.

\section{RES U L T S}

\section{Distribution of Infections and Pathogens by Hospital, Procedure, or Location Types}

From January 2011 through December 2014, a total of 365,490 HAIs were reported to NHSN from 4,515 hospitals. The relative proportions of HAIs varied by hospital type and size, with most HAIs reported from general acute care hospitals and hospitals with greater than 200 beds (Table 1). Overall, 408,151 pathogens were reported across all 4 HAI types; $38 \%$ of pathogens from CAUTIs, $37 \%$ from SSIs, $24 \%$ from CLABSIs, and $2 \%$ from VAPs (Table 2). Among the pathogens reported from SSIs, $51 \%$ were associated with abdominal surgeries (which includes colon surgeries, one of the procedures required by CMS' Hospital Inpatient QRP) and 23\% from orthopedic surgeries (Table 2). Each HAI was associated with an average of 1.1 reported pathogens.

TABLE 1. Characteristics of Hospitals Reporting HealthcareAssociated Infections (HAIs) to the National Healthcare Safety Network (NHSN), 2011-2014

\begin{tabular}{lcc}
\hline & $\begin{array}{c}\text { No. }(\%) \text { of hospitals } \\
\text { reporting }^{\mathrm{a}} \\
(\mathrm{n}=4,515)\end{array}$ & $\begin{array}{c}\text { No. }(\%) \text { of HAIs } \\
\text { reported } \\
(\mathrm{n}=365,490)\end{array}$ \\
Characteristic & & \\
\hline Hospital type & $3,180(70.4)$ & $321,487(88.0)$ \\
General & $508(11.3)$ & $23,827(6.5)$ \\
Long-term acute care & $342(7.6)$ & $1,772(0.5)$ \\
Critical access & $226(5.0)$ & $1,993(0.5)$ \\
Rehabilitation & $76(1.7)$ & $1,230(0.3)$ \\
Surgical & $74(1.6)$ & $6,899(1.9)$ \\
Children's & $28(0.6)$ & $886(0.2)$ \\
Military & $23(0.5)$ & $592(0.2)$ \\
Orthopedic & $18(0.4)$ & $4,163(1.1)$ \\
Oncology & $14(0.3)$ & $647(0.2)$ \\
Veterans Affairs & $10(0.2)$ & $1,052(0.3)$ \\
Women's and children's & $10(0.2)$ & $920(0.3)$ \\
Women's & $6(0.1)$ & $22(<0.1)$ \\
Psychiatric & & \\
Hospital bed size & $1,226(27.2)$ & $17,378(4.8)$ \\
$\leq 50$ & $1,924(42.6)$ & $68,080(18.6)$ \\
$51-200$ & $1,101(24.4)$ & $154,225(42.2)$ \\
$201-500$ & $264(5.8)$ & $125,807(34.4)$ \\
$\geq 501$ & &
\end{tabular}

${ }^{\mathrm{a}}$ The number that have reported at least 1 HAI used in this report in 2011-2014.

${ }^{\mathrm{b}}$ Does not include inpatient rehabilitation facilities reporting to NHSN as locations within enrolled acute care hospitals.
Pathogens from device-associated HAIs were reported from 17,600 individual locations within hospitals, including adult and pediatric critical care units, neonatal intensive care units, inpatient wards, step-down units, and others (Table 3). Fifty-three percent of pathogens from device-associated infections were reported from adult critical care units within acute care hospitals, of which the single largest contributing type was medical/surgical units (Table 3 ).

\section{Pathogen Distribution}

Overall, $87 \%$ of reported pathogens were from 1 of 15 pathogen groups (Table 4). The most common pathogens included E. coli (15.4\%), S. aureus (11.8\%), K. pneumoniae and K. oxytoca $(7.7 \%)$, coagulase-negative staphylococci $(7.7 \%)$, and Enterococcus faecalis (7.4\%) (Table 4). Each pathogen group was assigned a rank within each HAI type; the most prevalent pathogens were coagulase-negative staphylococci (CLABSI), E. coli (CAUTI), and S. aureus (VAP and SSI) (Table 4). Pathogen rankings would be impacted if some species were analyzed within their genus group. If all Enterococcus species were analyzed at the genus-level, this group would be considered the second most common pathogen across all HAI types, and the single most common pathogen among CLABSIs. If all Candida species were analyzed together, this pathogen group would be considered the fourth most common pathogen across all HAI types, and the second most common among CAUTIs.

For the 149,009 SSI pathogens reported, the pathogen distribution varied by surgery type (Table 5 ). S. aureus was the most prevalent SSI pathogen for most types of surgery, but E. coli was more prevalent in abdominal surgery SSIs. Enterococcus species were associated with almost 30\% of transplant surgery SSIs.

\section{Percent Tested and Percent Resistance}

The percent of pathogens tested for susceptibility varied by phenotype, HAI type, and period (Tables 6-9). Similar to the previous report, high susceptibility testing frequencies (ie, $>90 \%$ ) for almost all years included in this report and across all HAI types were reported for $S$. aureus testing to oxacillin/methicillin/cefoxitin, E. coli and $P$. aeruginosa testing to fluoroquinolones, $P$. aeruginosa testing to aminoglycosides, and Enterobacter spp. and $P$. aeruginosa testing to extendedspectrum cephalosporins (Tables 6-9). The percent of $P$. aeruginosa tested for aminoglycoside susceptibility in 2014 was at least $94 \%$ for all HAIs, which appears to be higher than values published in the previous report. ${ }^{2}$ Although the values varied by HAI type, hospitals continued to report low testing frequencies, especially in 2014 (range, 66.0\%-73.3\%), for K. oxytoca and K. pneumoniae, E. coli, and Enterobacter spp. to carbapenems (Tables 6-9).

The percent resistance for most pathogens was generally lower among SSIs compared with device-associated HAIs, and 
т A в LE 2. Types of Healthcare-Associated Infections (HAIs) and Surgical Site Infections (SSIs) Reported to the National Healthcare Safety Network (NHSN), 2011-2014

\begin{tabular}{lrr}
\hline Type of HAI & No. $(\%)$ of events reported $(\mathrm{n}=365,490)$ & No. $(\%)$ of pathogens reported $(\mathrm{n}=408,151)$ \\
\hline CLABSI & $85,994(23.5)$ & $96,532(23.7)$ \\
CAUTI & $138,283(37.8)$ & $153,805(37.7)$ \\
VAP ${ }^{\mathrm{a}, \mathrm{b}}$ & $8,133(2.2)$ & $8,805(2.2)$ \\
SSI $^{\mathrm{b}}$ & $133,080(36.4)$ & $149,009(36.5)$ \\
Type of Surgery & No. $(\%)$ of SSIs & No. (\%) of SSI pathogens \\
\hline Abdominal $^{\mathrm{c}}$ & $63,508(47.7)$ & $76,307(51.2)$ \\
Breast $^{\mathrm{d}}$ & $886(0.7)$ & $946(0.6)$ \\
Cardiac $^{\mathrm{e}}$ & $10,439(7.8)$ & $11,281(7.6)$ \\
Kidney $^{\mathrm{f}}$ & $251(0.2)$ & $285(0.2)$ \\
Neck $^{\mathrm{g}}$ & $146(0.1)$ & $212(0.1)$ \\
Neurological $^{\mathrm{h}}$ & $1,945(1.5)$ & $2,168(1.5)$ \\
Ob/Gyn $^{\mathrm{i}}$ & $22,231(16.7)$ & $20,725(13.9)$ \\
Orthopedic $^{\mathrm{j}}$ & $31,539(23.7)$ & $34,341(23.0)$ \\
Prostate $^{\mathrm{k}}$ & $53(<0.1)$ & $61(<0.1)$ \\
Transplant $^{1}$ & $644(0.5)$ & $815(0.5)$ \\
Vascular $^{\mathrm{m}}$ & $1,438(1.1)$ & $1,868(1.3)$ \\
\hline
\end{tabular}

NOTE. CAUTI, catheter-associated urinary tract infection; CLABSI, central line-associated bloodstream infection; $\mathrm{Ob} / \mathrm{Gyn}$, obstetrical and gynecological; SSI, surgical site infection; VAP, ventilator-associated pneumonia.

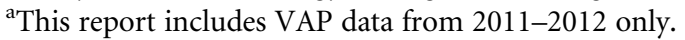

${ }^{b}$ SSI and VAP events can be reported without a pathogen. Total of 29,469 events (8.1\%) were reported without a pathogen ( $\mathrm{SSI}=28,227$ events, $\mathrm{VAP}=1,242$ events).

${ }^{\mathrm{c}}$ Appendectomy, bile duct, liver, or pancreatic surgery, gallbladder surgery, colon surgery, gastric surgery, herniorrhaphy, small bowel surgery, spleen surgery, abdominal surgery, and rectal surgery.

${ }^{\mathrm{d}}$ Breast surgery.

${ }^{\mathrm{e}}$ Cardiac surgery, coronary artery bypass graft with chest incision with or without donor incision, pacemaker surgery, and thoracic surgery.

${ }^{\mathrm{f}}$ Kidney surgery.

${ }^{\mathrm{g}} \mathrm{Neck}$ surgery and thyroid and/or parathyroid surgery.

${ }^{\mathrm{h}}$ Craniotomy and ventricular shunt.

${ }^{\mathrm{i} C}$ Cesarean delivery, abdominal hysterectomy, ovarian surgery, and vaginal hysterectomy.

jOpen reduction of fracture, hip prosthesis, knee prosthesis, limb amputation, spinal fusion, refusion of spine, and laminectomy.

${ }^{\mathrm{k}}$ Prostate surgery.

${ }^{1}$ Heart transplant, kidney transplant, and liver transplant.

${ }^{\mathrm{m}}$ Abdominal aortic aneurysm repair, shunt for dialysis, carotid endarterectomy, and peripheral vascular bypass surgery.

differed only slightly between device-associated infection types. Acinetobacter spp. resistance to carbapenems (64.0\%) and multidrug-resistance (69.1\%) in 2014 appeared highest in CAUTI (Table 7). In addition, the magnitude of the resistance percentage appears to be changing for certain phenotypes. For all HAIs, the proportions of Acinetobacter pathogens with the 2 resistant phenotypes were noticeably lower in 2014 compared with 2011. A lower proportion of Klebsiella pathogens among CLABSIs in 2014 were resistant to extended-spectrum cephalosporins or identified as multidrug-resistant compared with 2011-2013 (Table 6). However, a higher proportion of E. coli pathogens were reported with fluoroquinolone resistance each year in 2011-2014 in CLABSIs, and more frequent reporting of multidrug-resistance and extended-spectrum cephalosporin resistance among CLABSI and CAUTI E. coli pathogens was seen in 2013 and 2014 compared with earlier years (Tables 6 and 7).
The proportion of Enterobacteriaceae that were resistant to carbapenems (CRE) was highest among CLABSI pathogens, particularly K. pneumoniae and K. oxytoca (Table 10). In 2014, $7.1 \%$ of all tested Enterobacteriaceae CLABSI pathogens were resistant to carbapenems, whereas $4.0 \%$ and $1.8 \%$ of CAUTI and SSI Enterobacteriaceae pathogens were carbapenem-resistant (Table 10). Applying the updated CRE definition resulted in a small decrease in the percent resistance and an increase in the percent of pathogens tested for susceptibility (Table 10).

\section{DISCUSSION}

These data underscore the broad reach of the antimicrobial resistance problem and the challenges confronting clinicians, healthcare organizations, public health agencies, and patients throughout the nation. This is the first NHSN antimicrobial 


\begin{tabular}{|c|c|c|c|c|c|}
\hline \multirow[b]{2}{*}{ Location } & \multirow{2}{*}{$\begin{array}{l}\text { No. }(\%) \text { of } \\
\text { units reporting } \\
(\mathrm{n}=17,600)\end{array}$} & \multicolumn{4}{|c|}{ No. (\%) of pathogens by HAI type } \\
\hline & & $\begin{array}{c}\text { Overall } \\
(\mathrm{n}=259,142)\end{array}$ & $\begin{array}{c}\text { CLABSI } \\
(\mathrm{n}=96,532)\end{array}$ & $\begin{array}{c}\text { CAUTI } \\
(\mathrm{n}=153,805) \\
\end{array}$ & $\begin{array}{c}\mathrm{VAP}^{\mathrm{a}} \\
(\mathrm{n}=8,805) \\
\end{array}$ \\
\hline \multicolumn{6}{|l|}{ Acute care hospitals } \\
\hline \multicolumn{6}{|l|}{ Critical care units } \\
\hline Adult medical & $748(4.3)$ & $21,758(8.4)$ & $6,333(6.6)$ & $14,659(9.5)$ & $766(8.7)$ \\
\hline Adult medical/surgical & $2,807(15.9)$ & $54,453(21.0)$ & $16,943(17.6)$ & $34,773(22.6)$ & $2,737(31.1)$ \\
\hline All other adult critical care & $1,871(10.6)$ & $59,851(23.1)$ & $15,046(15.6)$ & $40,909(26.6)$ & $3,896(44.2)$ \\
\hline Pediatric critical care & $376(2.1)$ & $5,812(2.2)$ & $3,544(3.7)$ & $1,960(1.3)$ & $308(3.5)$ \\
\hline Neonatal intensive care $(\mathrm{NICU})^{\mathrm{b}}$ & $791(4.5)$ & $8,483(3.3)$ & $7,844(8.1)$ & $\ldots$ & $639(7.3)$ \\
\hline \multicolumn{6}{|l|}{ Inpatient wards } \\
\hline Adult medical ward & $1,484(8.4)$ & $11,872(4.6)$ & $5,139(5.3)$ & $6,729(4.4)$ & $4(<0.1)$ \\
\hline Adult medical/surgical ward & $2,977(16.9)$ & $20,460(7.9)$ & $7,886(8.2)$ & $12,526(8.1)$ & $48(0.5)$ \\
\hline All other adult ward and step-down & $3,648(20.7)$ & $27,967(10.8)$ & $8,867(9.2)$ & $18,990(12.3)$ & $110(1.2)$ \\
\hline Pediatric ward and step-down & $379(2.2)$ & $2,092(0.8)$ & $1,756(1.8)$ & $333(0.2)$ & $3(<0.1)$ \\
\hline \multicolumn{6}{|l|}{ Other locations } \\
\hline Oncology $^{c}$ & $616(3.5)$ & $13,628(5.3)$ & $11,399(11.8)$ & $2,222(1.4)$ & $7(0.1)$ \\
\hline Chronic care & $54(0.3)$ & $507(0.2)$ & $166(0.2)$ & $309(0.2)$ & $32(0.4)$ \\
\hline \multicolumn{6}{|l|}{ Long-term acute care (LTAC) hospitals ${ }^{\mathrm{d}}$} \\
\hline LTAC critical care ${ }^{\mathrm{e}}$ & $91(0.5)$ & $1,642(0.6)$ & $748(0.8)$ & $885(0.6)$ & $9(0.1)$ \\
\hline LTAC ward $^{\mathrm{f}}$ & $705(4.0)$ & $25,185(9.7)$ & $10,410(10.8)$ & $14,529(9.4)$ & $246(2.8)$ \\
\hline \multicolumn{6}{|l|}{ Inpatient rehabilitation facilities (IRF) ${ }^{\mathrm{d}}$} \\
\hline Free-standing & $312(1.8)$ & $2,242(0.9)$ & $126(0.1)$ & $2,116(1.4)$ & $\ldots$ \\
\hline Within a healthcare facility ${ }^{\mathrm{g}}$ & $15(0.1)$ & $74(<0.1)$ & $8(<0.1)$ & $66(<0.1)$ & $\ldots$ \\
\hline Location within acute care hospital $^{\mathrm{h}}$ & $726(4.1)$ & $3,116(1.2)$ & $317(0.3)$ & $2,799(1.8)$ & $\ldots$ \\
\hline
\end{tabular}

NOTE. CAUTI, catheter-associated urinary tract infection; CLABSI, central line-associated bloodstream infection; HAI, healthcare-associated infection; VAP, ventilatorassociated pneumonia.

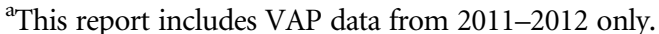

${ }^{\mathrm{b}}$ NICU locations included are those classified by NHSN location codes as level II/III and level III neonatal critical care areas.

A level II/III neonatal critical care area is defined by NHSN as a combined nursery housing both level II and III newborns and infants. A level III neonatal critical care area is defined by NHSN as a hospital NICU organized with personnel and equipment to provide continuous life support and comprehensive care for extremely high-risk newborn infants and those with complex and critical illness.

${ }^{\mathrm{c}}$ Includes Oncology locations of all acuity levels (critical care, ward, and step-down) located within general acute care hospitals and oncology hospitals.

${ }^{\mathrm{d}}$ LTAC and IRF Quality Reporting Programs began in October 2012, and therefore data reported from these facilities may not be inclusive of the entire 4-year period shown in the table.

${ }^{\mathrm{e}}$ Includes adult and pediatric LTAC critical care units.

Includes adult and pediatric LTAC wards.

${ }^{\mathrm{g}}$ IRFs that function as part of a larger healthcare facility.

${ }^{\mathrm{h}}$ Rehabilitation wards located within enrolled acute care hospitals and defined as IRFs per the Centers for Medicare and Medicaid Services IRF Quality Reporting Program. 
тав LE 4. Distribution and Rank Order of Pathogens Frequently Reported to the National Healthcare Safety Network (NHSN), by Type of Healthcare-Associated Infection (HAI), $2011-2014$

\begin{tabular}{|c|c|c|c|c|c|c|c|c|c|c|}
\hline \multirow[b]{2}{*}{ Pathogen } & \multicolumn{2}{|l|}{ Overall } & \multicolumn{2}{|l|}{ CLABSI } & \multicolumn{2}{|l|}{ CAUTI } & \multicolumn{2}{|l|}{ VAP $^{a}$} & \multicolumn{2}{|l|}{ SSI } \\
\hline & $\begin{array}{l}\text { No. }(\%) \text { of } \\
\text { pathogens }\end{array}$ & $\operatorname{Rank}^{\mathrm{b}}$ & $\begin{array}{l}\text { No. }(\%) \text { of } \\
\text { pathogens }\end{array}$ & $\operatorname{Rank}^{\mathrm{b}}$ & $\begin{array}{l}\text { No. }(\%) \text { of } \\
\text { pathogens }\end{array}$ & $\operatorname{Rank}^{\mathrm{b}}$ & $\begin{array}{c}\text { No. (\%) of } \\
\text { pathogens }\end{array}$ & $\operatorname{Rank}^{\mathrm{b}}$ & $\begin{array}{c}\text { No. (\%) of } \\
\text { pathogens }\end{array}$ & $\operatorname{Rank}^{\mathrm{b}}$ \\
\hline Escherichia coli & $62,904(15.4)$ & 1 & $5,193(5.4)$ & 7 & $36,806(23.9)$ & 1 & $476(5.4)$ & 6 & $20,429(13.7)$ & 2 \\
\hline Staphylococcus aureus & $48,302(11.8)$ & 2 & $12,706(13.2)$ & 2 & $2,515(1.6)$ & 14 & $2,179(24.7)$ & 1 & $30,902(20.7)$ & 1 \\
\hline Klebsiella (pneumoniae/oxytoca) & $31,498(7.7)$ & 3 & $8,062(8.4)$ & 4 & $15,471(10.1)$ & 4 & $898(10.2)$ & 3 & $7,067(4.7)$ & 6 \\
\hline Coagulase-negative staphylococci ${ }^{\mathrm{c}}$ & $31,361(7.7)$ & 4 & $15,794(16.4)$ & 1 & $3,696(2.4)$ & 13 & $72(0.8)$ & 13 & $11,799(7.9)$ & 3 \\
\hline Enterococcus faecalis ${ }^{\mathrm{d}}$ & $30,034(7.4)$ & 5 & $8,118(8.4)$ & 3 & $10,728(7.0)$ & 5 & $32(0.4)$ & 21 & $11,156(7.5)$ & 4 \\
\hline Pseudomonas aeruginosa & $29,636(7.3)$ & 6 & $3,881(4.0)$ & 10 & $15,848(10.3)$ & 3 & $1,449(16.5)$ & 2 & $8,458(5.7)$ & 5 \\
\hline Candida albicans ${ }^{\mathrm{d}}$ & $27,231(6.7)$ & 7 & $5,761(6.0)$ & 6 & $17,926(11.7)$ & 2 & $193(2.2)$ & 10 & $3,351(2.2)$ & 12 \\
\hline Enterobacter $\mathrm{spp}^{\mathrm{c}}$ & $17,235(4.2)$ & 8 & $4,204(4.4)$ & 9 & $5,689(3.7)$ & 9 & $727(8.3)$ & 4 & $6,615(4.4)$ & 8 \\
\hline Enterococcus faecium ${ }^{\mathrm{d}}$ & $14,942(3.7)$ & 9 & $6,567(6.8)$ & 5 & $4,212(2.7)$ & 11 & $23(0.3)$ & 24 & $4,140(2.8)$ & 11 \\
\hline Other Enterococcus spp. ${ }^{\mathrm{d}}$ & $14,694(3.6)$ & 10 & $1,974(2.0)$ & 14 & $6,291(4.1)$ & 7 & $19(0.2)$ & 27 & $6,410(4.3)$ & 9 \\
\hline Proteus spp. ${ }^{c}$ & $11,249(2.8)$ & 11 & $820(0.8)$ & 17 & $6,108(4.0)$ & 8 & $125(1.4)$ & 12 & $4,196(2.8)$ & 10 \\
\hline Yeast $\operatorname{NOS}^{\mathrm{e}}$ & $10,811(2.6)$ & 12 & $763(0.8)$ & 18 & $9,443(6.1)$ & 6 & $54(0.6)$ & 16 & $551(0.4)$ & 25 \\
\hline Other Candida spp. ${ }^{\mathrm{d}}$ & $10,641(2.6)$ & 13 & $4,730(4.9)$ & 8 & $5,178(3.4)$ & 10 & $37(0.4)$ & 19 & $696(0.5)$ & 19 \\
\hline Candida glabrata & $8,121(2.0)$ & 14 & $3,314(3.4)$ & 11 & $4,121(2.7)$ & 12 & $12(0.1)$ & 33 & $674(0.5)$ & 20 \\
\hline Bacteroides spp. & $7,560(1.9)$ & 15 & $515(0.5)$ & 19 & $2(<0.1)$ & 130 & $2(<0.1)$ & 72 & $7,041(4.7)$ & 7 \\
\hline Other pathogen & $51,932(12.7)$ & & $14,130(14.6)$ & & $9,771(6.4)$ & & $2,507(28.5)$ & & $25,524(17.1)$ & \\
\hline Total & $408,151(100)$ & & $96,532(100)$ & & $153,805(100)$ & & $8,805(100)$ & & $149,009(100)$ & \\
\hline
\end{tabular}

NOTE. CAUTI, catheter-associated urinary tract infection; CLABSI, central line-associated bloodstream infection; NOS, not otherwise specified; SSI, surgical site infection; VAP, ventilatorassociated pneumonia.

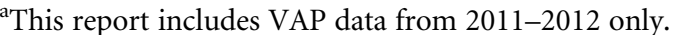

${ }^{\mathrm{b}}$ The 15 most common pathogens are listed in this table and ranked according to how frequently they were reported to NHSN. The rankings were established based on all pathogens reported.

'Among all HAIs, the following species were frequently reported but considered part of a larger pathogen group for this table: Staphylococcus epidermidis (12,562 pathogens reported), Enterobacter cloacae (11,269), and Proteus mirabilis (10,559).

${ }^{\mathrm{a}}$ For informational purposes, select pathogens were also categorized at the combined genus-level with the following results: All Enterococcus species (E. faecalis, E. faecium, and other species) were ranked Overall (2), CLABSI (1), CAUTI (3), VAP (11), SSI (2) and all Candida species (C. albicans, C. glabrata, and other species) were ranked Overall (4), CLABSI (3), CAUTI (2), VAP (9), SSI (10).

'Other non-Candida yeast, or yeast not otherwise specified. 


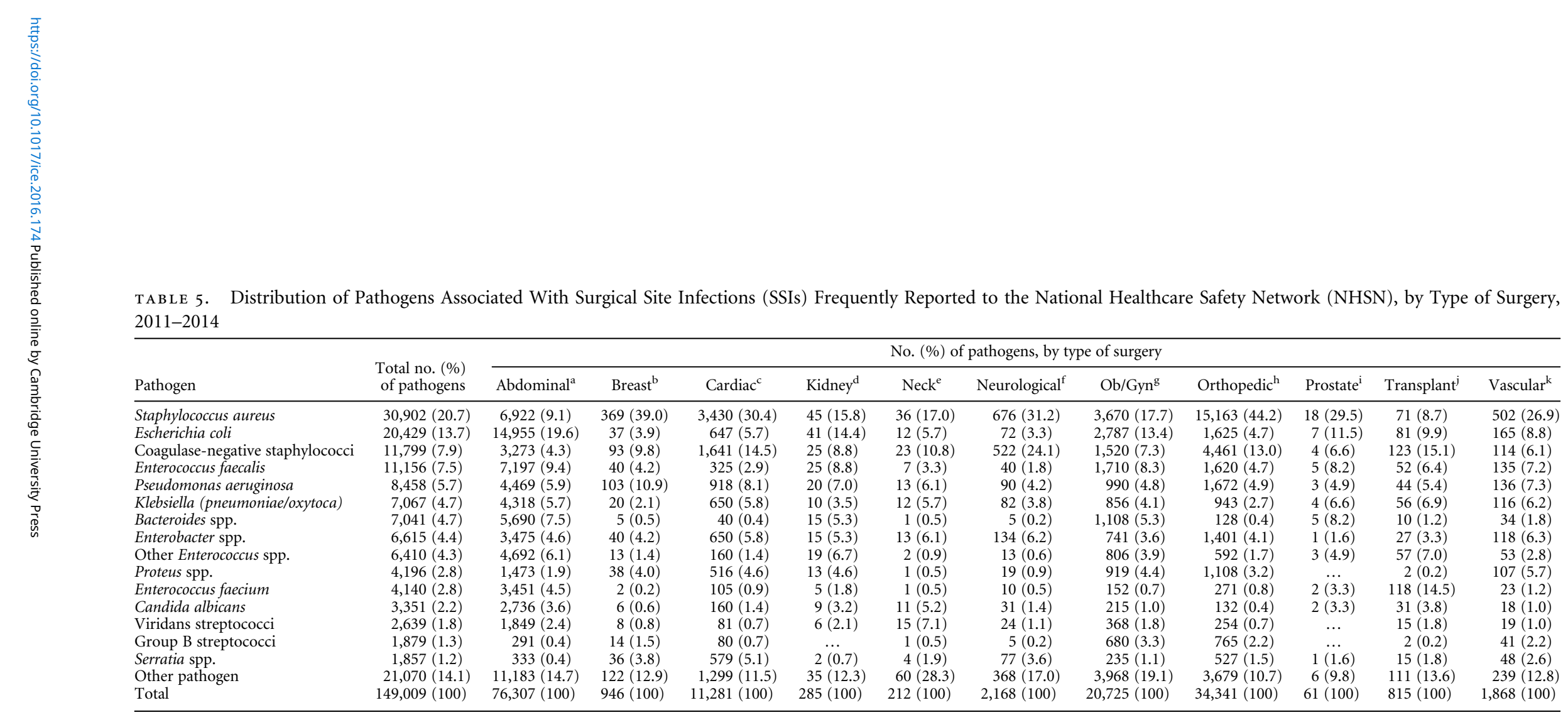
2011-2014

NOTE. Ob/Gyn, obstetrical and gynecological.

${ }^{a}$ Appendectomy, bile duct, liver, or pancreatic surgery, gallbladder surgery, colon surgery, gastric surgery, herniorrhaphy, small bowel surgery, spleen surgery, abdominal surgery, and rectal surgery.

${ }^{\mathrm{b}}$ Breast surgery.

${ }^{\mathrm{c} C a r d i a c}$ surgery, coronary artery bypass graft with chest incision with or without donor incision, pacemaker surgery, and thoracic surgery.

${ }^{\mathrm{d}}$ Kidney surgery.

${ }^{\mathrm{e}} \mathrm{Neck}$ surgery and thyroid and/or parathyroid surgery.

${ }^{\mathrm{f}}$ Craniotomy and ventricular shunt.

${ }^{\mathrm{g} C}$ Cesarean delivery, abdominal hysterectomy, ovarian surgery, and vaginal hysterectomy.

${ }^{\mathrm{h}}$ Open reduction of fracture, hip prosthesis, knee prosthesis, limb amputation, spinal fusion, refusion of spine, and laminectomy.

${ }^{\mathrm{i}}$ Prostate surgery.

${ }^{j}$ Heart transplant, kidney transplant, and liver transplant.

${ }^{\mathrm{k}}$ Abdominal aortic aneurysm repair, shunt for dialysis, carotid endarterectomy, and peripheral vascular bypass surgery. 
тав вE 6. Percent of Pathogens Reported From Central Line-Associated Bloodstream Infections (CLABSIs) That Tested Resistant to Selected Antimicrobial Agents, by Period, $2011-2014$

\begin{tabular}{|c|c|c|c|c|c|c|c|c|c|c|c|c|}
\hline \multirow[b]{2}{*}{ Pathogen, antimicrobial } & \multicolumn{3}{|c|}{2011} & \multicolumn{3}{|c|}{2012} & \multicolumn{3}{|c|}{2013} & \multicolumn{3}{|c|}{2014} \\
\hline & $\begin{array}{l}\text { No. of isolates } \\
\text { reported }\end{array}$ & $\begin{array}{c}\% \text { of isolates }_{\text {tested }}^{\mathrm{a}}\end{array}$ & $\begin{array}{c}\% \\
\text { Resistance }\end{array}$ & $\begin{array}{l}\text { No. of isolates } \\
\text { reported }\end{array}$ & $\begin{array}{c}\% \text { of isolates } \\
\text { tested }^{\mathrm{a}}\end{array}$ & $\begin{array}{c}\% \\
\text { Resistance }\end{array}$ & $\begin{array}{l}\text { No. of isolates } \\
\text { reported }\end{array}$ & $\begin{array}{l}\% \text { of isolates } \\
\text { tested }^{\mathrm{a}}\end{array}$ & $\begin{array}{c}\% \\
\text { Resistance }\end{array}$ & $\begin{array}{l}\text { No. of isolates } \\
\text { reported }\end{array}$ & $\begin{array}{l}\% \text { of isolates } \\
\text { tested }^{\mathrm{a}}\end{array}$ & $\begin{array}{c}\% \\
\text { Resistance }\end{array}$ \\
\hline Staphylococcus aureus & 3,022 & & & 3,087 & & & 3,358 & & & 3,239 & & \\
\hline OX/METH/CEFOX & & 93.3 & 52.6 & & 92.6 & 51.1 & & 91.0 & 52.3 & & 90.3 & 50.7 \\
\hline Enterococcus spp. & & & & & & & & & & & & \\
\hline E. faecium & 1,550 & & & 1,532 & & & 1,756 & & & 1,729 & & \\
\hline VAN & & 95.7 & 83.8 & & 96.2 & 83.3 & & 94.3 & 83.0 & & 94.8 & 82.2 \\
\hline E. faecalis & 1,984 & & & 2,080 & & & 2,107 & & & 1,947 & & \\
\hline VAN & & 93.5 & 9.9 & & 93.2 & 10.1 & & 93.5 & 9.3 & & 93.9 & 9.8 \\
\hline Klebsiella (pneumoniae/oxytoca) & 1,851 & & & 1,936 & & & 2,075 & & & 2,200 & & \\
\hline ESC4 & & 85.6 & 28.3 & & 84.9 & 28.1 & & 85.8 & 28.5 & & 85.1 & 24.1 \\
\hline Carbapenems & & 74.8 & 11.3 & & 75.8 & 13.0 & & 74.8 & 13.1 & & 73.3 & 10.9 \\
\hline MDR1 & & 90.2 & 20.9 & & 91.6 & 20.3 & & 92.9 & 20.3 & & 92.6 & 17.2 \\
\hline Escherichia coli & 956 & & & 1,167 & & & 1,475 & & & 1,595 & & \\
\hline ESC4 & & 85.1 & 19.7 & & 83.5 & 22.2 & & 84.9 & 24.4 & & 84.6 & 22.2 \\
\hline FQ3 & & 91.6 & 41.1 & & 90.8 & 42.5 & & 89.4 & 47.8 & & 90.1 & 49.3 \\
\hline Carbapenems & & 74.4 & 1.3 & & 73.2 & 1.3 & & 71.2 & 2.1 & & 70.9 & 1.9 \\
\hline MDR1 & & 90.2 & 11.1 & & 90.7 & 13.8 & & 92.1 & 14.9 & & 90.9 & 14.1 \\
\hline Enterobacter spp. & 1,000 & & & 1,029 & & & 1,106 & & & 1,069 & & \\
\hline ESC4 & & 93.5 & 37.3 & & 91.6 & 38.2 & & 91.9 & 37.7 & & 89.8 & 36.1 \\
\hline Carbapenems & & 76.7 & 3.0 & & 74.2 & 5.2 & & 72.8 & 6.2 & & 70.7 & 6.6 \\
\hline MDR1 & & 93.9 & 8.1 & & 93.1 & 10.0 & & 93.2 & 10.4 & & 92.2 & 9.5 \\
\hline Pseudomonas aeruginosa & 888 & & & 877 & & & 1,100 & & & 1,016 & & \\
\hline AMINOS & & 92.5 & 22.0 & & 96.9 & 17.5 & & 94.5 & 20.5 & & 94.0 & 17.2 \\
\hline ESC2 & & 92.1 & 27.1 & & 95.2 & 23.2 & & 92.5 & 26.6 & & 92.7 & 24.2 \\
\hline FQ2 & & 93.8 & 33.1 & & 92.9 & 28.3 & & 90.5 & 31.4 & & 92.2 & 30.2 \\
\hline Carbapenems & & 83.8 & 28.4 & & 84.3 & 23.7 & & 83.1 & 25.4 & & 80.9 & 25.8 \\
\hline PIP/ PIPTAZ & & 81.0 & 19.9 & & 82.3 & 17.9 & & 84.6 & 19.0 & & 87.2 & 18.4 \\
\hline MDR2 & & 95.0 & 21.7 & & 96.9 & 16.7 & & 93.9 & 19.0 & & 94.4 & 17.9 \\
\hline Acinetobacter spp. & 544 & & & 572 & & & 538 & & & 495 & & \\
\hline Carbapenems & & 83.3 & 57.2 & & 82.7 & 49.5 & & 79.7 & 53.1 & & 76.4 & 46.6 \\
\hline MDR3 & & 96.3 & 60.9 & & 95.3 & 51.6 & & 95.2 & 52.7 & & 92.9 & 43.7 \\
\hline
\end{tabular}

NOTE. OX/METH/CEFOX, oxacillin/methicillin/cefoxitin; VAN, vancomycin; ESC4, extended-spectrum cephalosporin (cefepime, cefotaxime, ceftazidime, ceftriaxone); Carbapenems (imipenem, meropenem, doripenem); MDR1, multidrug-resistance (must test either intermediate [I] or resistant [R] to at least 1 drug in 3 of the 5 following classes [ESC4, FQ3, AMINO, carbapenems, \& PIP/PIPTAZ]); FQ3, fluoroquinolones (ciprofloxacin, levofloxacin, moxifloxacin); AMINOS, aminoglycosides (amikacin, gentamicin, tobramycin); ESC2, extendedspectrum cephalosporin (cefepime, ceftazidime); FQ2, fluoroquinolones (ciprofloxacin, levofloxacin); PIP, piperacillin; PIPTAZ, piperacillin/tazobactam; MDR2, multidrug-resistance (must test either I or R to at least 1 drug in 3 of the 5 following classes [ESC2, FQ2, AMINOS, carbapenems, \& PIP/PIPTAZ]); MDR3, multidrug-resistance (must test either I or R to at least 1 drug in 3 of the 6 following classes [ESC4, FQ2, AMINOS, carbapenems, PIP/PIPTAZ \& ampicillin/sulbactam]).

${ }^{a}$ If the percent of isolates tested is less than $70 \%$, caution should be used when interpreting the percent resistance. 
TA B LE 7. Percent of Pathogens Reported From Catheter-Associated Urinary Tract Infections (CAUTIs) That Tested Resistant to Selected Antimicrobial Agents, by Period, 20112014

\begin{tabular}{|c|c|c|c|c|c|c|c|c|c|c|c|c|}
\hline \multirow[b]{2}{*}{ Pathogen, antimicrobial } & \multicolumn{3}{|c|}{2011} & \multicolumn{3}{|c|}{2012} & \multicolumn{3}{|c|}{2013} & \multicolumn{3}{|c|}{2014} \\
\hline & $\begin{array}{l}\text { No. of isolates } \\
\text { reported }\end{array}$ & $\begin{array}{l}\% \text { of isolates } \\
\text { tested }^{\mathrm{a}}\end{array}$ & $\begin{array}{c}\% \\
\text { Resistance }\end{array}$ & $\begin{array}{l}\text { No. of isolates } \\
\text { reported }\end{array}$ & $\begin{array}{l}\% \text { of isolates } \\
\text { tested }^{\mathrm{a}}\end{array}$ & $\begin{array}{c}\% \\
\text { Resistance }\end{array}$ & $\begin{array}{l}\text { No. of isolates } \\
\text { reported }\end{array}$ & $\begin{array}{l}\% \text { of isolates } \\
\text { tested }^{\mathrm{a}}\end{array}$ & $\begin{array}{c}\% \\
\text { Resistance }\end{array}$ & $\begin{array}{l}\text { No. of isolates } \\
\text { reported }\end{array}$ & $\begin{array}{l}\% \text { of isolates } \\
\text { tested }^{\mathrm{a}}\end{array}$ & $\begin{array}{c}\% \\
\text { Resistance }\end{array}$ \\
\hline Staphylococcus aureus & 328 & & & 665 & & & 742 & & & 780 & & \\
\hline OX/METH/CEFOX & & 96.6 & 55.8 & & 92.9 & 56.8 & & 92.7 & 55.5 & & 92.9 & 52.0 \\
\hline Enterococcus spp. & & & & & & & & & & & & \\
\hline E. faecium & 598 & & & 1,148 & & & 1,255 & & & 1,211 & & \\
\hline VAN & & 96.2 & 83.8 & & 96.6 & 86.0 & & 96.5 & 86.2 & & 96.1 & 85.1 \\
\hline E. faecalis & 1,460 & & & 2,911 & & & 3,112 & & & 3,245 & & \\
\hline VAN & & 94.1 & 7.1 & & 92.2 & 7.4 & & 92.5 & 9.1 & & 93.6 & 8.0 \\
\hline Klebsiella (pneumoniae/oxytoca) & 2,035 & & & 4,170 & & & 4,541 & & & 4,725 & & \\
\hline ESC4 & & 84.2 & 21.8 & & 84.6 & 20.6 & & 85.3 & 23.9 & & 84.6 & 22.5 \\
\hline Carbapenems & & 67.3 & 10.7 & & 71.8 & 9.1 & & 71.1 & 10.9 & & 68.8 & 9.5 \\
\hline MDR1 & & 90.3 & 14.8 & & 91.2 & 15.0 & & 93.5 & 17.2 & & 93.2 & 14.6 \\
\hline Escherichia coli & 4,826 & & & 10,512 & & & 10,628 & & & 10,840 & & \\
\hline ESC4 & & 82.8 & 12.9 & & 82.6 & 12.8 & & 84.0 & 15.5 & & 84.0 & 16.1 \\
\hline FQ3 & & 96.3 & 32.7 & & 96.1 & 31.0 & & 96.2 & 35.4 & & 96.3 & 34.8 \\
\hline Carbapenems & & 63.8 & 1.2 & & 66.2 & 0.8 & & 67.5 & 1.0 & & 66.6 & 1.1 \\
\hline MDR1 & & 87.8 & 5.5 & & 89.4 & 6.2 & & 92.8 & 8.1 & & 93.7 & 8.0 \\
\hline Enterobacter spp. & 727 & & & 1,614 & & & 1,707 & & & 1,641 & & \\
\hline ESC4 & & 93.1 & 40.6 & & 92.7 & 39.5 & & 91.9 & 38.8 & & 92.9 & 40.5 \\
\hline Carbapenems & & 67.1 & 3.9 & & 68.7 & 4.2 & & 67.9 & 7.1 & & 70.7 & 6.5 \\
\hline MDR1 & & 92.0 & 10.5 & & 95.2 & 9.4 & & 94.8 & 10.5 & & 95.2 & 11.2 \\
\hline Pseudomonas aeruginosa & 2,023 & & & 4,320 & & & 4,848 & & & 4,657 & & \\
\hline AMINOS & & 94.4 & 25.1 & & 97.8 & 19.9 & & 97.6 & 22.4 & & 97.6 & 21.1 \\
\hline ESC2 & & 95.9 & 25.0 & & 96.0 & 22.3 & & 95.6 & 24.0 & & 96.3 & 22.5 \\
\hline $\mathrm{FQ} 2$ & & 96.6 & 34.5 & & 96.7 & 31.2 & & 96.4 & 34.0 & & 96.7 & 32.6 \\
\hline Carbapenems & & 78.6 & 22.3 & & 80.8 & 20.9 & & 82.1 & 24.8 & & 80.6 & 23.9 \\
\hline PIP/ PIPTAZ & & 77.4 & 16.5 & & 77.2 & 15.1 & & 86.8 & 15.8 & & 89.5 & 15.5 \\
\hline MDR2 & & 97.2 & 18.6 & & 97.9 & 16.7 & & 97.5 & 19.3 & & 97.6 & 17.7 \\
\hline Acinetobacter spp. & 158 & & & 294 & & & 345 & & & 276 & & \\
\hline Carbapenems & & 73.4 & 69.0 & & 75.5 & 57.7 & & 78.8 & 66.5 & & 81.5 & 64.0 \\
\hline MDR3 & & 98.7 & 75.6 & & 96.9 & 64.6 & & 96.8 & 73.1 & & 96.0 & 69.1 \\
\hline
\end{tabular}

NOTE. OX/METH/CEFOX, oxacillin/methicillin/cefoxitin; VAN, vancomycin; ESC4, extended-spectrum cephalosporin (cefepime, cefotaxime, ceftazidime, ceftriaxone); Carbapenems (imipenem, meropenem, doripenem); MDR1, multidrug-resistance (must test either intermediate [I] or resistant [R] to at least 1 drug in 3 of the 5 following classes [ESC4, FQ3, AMINO, carbapenems, \& PIP/PIPTAZ]); FQ3, fluoroquinolones (ciprofloxacin, levofloxacin, moxifloxacin); AMINOS, aminoglycosides (amikacin, gentamicin, tobramycin); ESC2, extendedspectrum cephalosporin (cefepime, ceftazidime); FQ2, fluoroquinolones (ciprofloxacin, levofloxacin); PIP, piperacillin; PIPTAZ, piperacillin/tazobactam; MDR2, multidrug-resistance (must test either I or R to at least 1 drug in 3 of the 5 following classes [ESC2, FQ2, AMINOS, carbapenems, \& PIP/PIPTAZ]); MDR3, multidrug-resistance (must test either I or R to at least 1 drug in 3 of the 6 following classes [ESC4, FQ2, AMINOS, carbapenems, PIP/PIPTAZ, \& ampicillin/sulbactam]).

${ }^{a} \mathrm{f}$ the percent of isolates tested is less than $70 \%$, caution should be used when interpreting the percent resistance. 
table 8. Percent of Pathogens Reported From Ventilator-Associated Pneumonias (VAPs) That Tested Resistant to Selected Antimicrobial Agents, by Period, 2011-2012

\begin{tabular}{|c|c|c|c|c|c|c|}
\hline \multirow[b]{2}{*}{ Pathogen, antimicrobial } & \multicolumn{3}{|c|}{$2011^{\mathrm{a}}$} & \multicolumn{3}{|c|}{$2012^{\mathrm{a}}$} \\
\hline & $\begin{array}{l}\text { No. of isolates } \\
\text { reported }\end{array}$ & $\begin{array}{c}\% \text { of isolates } \\
\text { tested }^{\mathrm{b}}\end{array}$ & $\begin{array}{c}\% \\
\text { Resistance }^{c}\end{array}$ & $\begin{array}{l}\text { No. of isolates } \\
\text { reported }\end{array}$ & $\begin{array}{c}\% \text { of isolates } \\
\text { tested }^{\mathrm{b}}\end{array}$ & $\begin{array}{c}\% \\
\text { Resistance }^{\mathrm{c}}\end{array}$ \\
\hline Staphylococcus aureus & 1,062 & & & 1,117 & & \\
\hline OX/METH/CEFOX & & 96.5 & 46.1 & & 96.5 & 42.4 \\
\hline \multicolumn{7}{|l|}{ Enterococcus spp. } \\
\hline E. faecium & 13 & & & 10 & & \\
\hline VAN & & 84.6 & $\ldots$ & & 100.0 & $\ldots$ \\
\hline E. faecalis & 14 & & & 18 & & \\
\hline VAN & & 78.6 & $\ldots$ & & 94.4 & $\ldots$ \\
\hline Klebsiella (pneumoniae/oxytoca) & 424 & & & 474 & & \\
\hline ESC4 & & 88.4 & 23.2 & & 86.3 & 21.0 \\
\hline Carbapenems & & 75.9 & 11.5 & & 75.1 & 10.1 \\
\hline MDR1 & & 93.6 & 15.9 & & 93.9 & 12.8 \\
\hline Escherichia coli & 219 & & & 257 & & \\
\hline ESC4 & & 88.1 & 15.0 & & 81.7 & 16.7 \\
\hline FQ3 & & 96.3 & 38.9 & & 93.4 & 30.8 \\
\hline Carbapenems & & 79.5 & 1.1 & & 69.3 & 2.2 \\
\hline MDR1 & & 94.5 & 7.7 & & 92.6 & 9.7 \\
\hline Enterobacter spp. & 338 & & & 389 & & \\
\hline ESC4 & & 95.6 & 30.0 & & 93.6 & 26.9 \\
\hline Carbapenems & & 76.6 & 1.9 & & 72.2 & 3.2 \\
\hline MDR1 & & 95.6 & 5.3 & & 96.1 & 2.9 \\
\hline Pseudomonas aeruginosa & 702 & & & 747 & & \\
\hline AMINOS & & 94.7 & 23.3 & & 96.9 & 18.2 \\
\hline ESC2 & & 96.6 & 29.4 & & 94.8 & 25.7 \\
\hline FQ2 & & 96.3 & 31.8 & & 94.0 & 31.9 \\
\hline Carbapenems & & 87.3 & 27.6 & & 81.7 & 28.4 \\
\hline PIP/ PIPTAZ & & 83.3 & 19.1 & & 81.5 & 19.4 \\
\hline MDR2 & & 98.1 & 20.8 & & 96.4 & 19.9 \\
\hline Acinetobacter spp. & 287 & & & 252 & & \\
\hline Carbapenems & & 85.0 & 63.5 & & 82.9 & 55.5 \\
\hline MDR3 & & 98.6 & 63.3 & & 98.8 & 53.8 \\
\hline
\end{tabular}

NOTE. OX/METH/CEFOX, oxacillin/methicillin/cefoxitin; VAN, vancomycin; ESC4, extended-spectrum cephalosporin (cefepime, cefotaxime, ceftazidime, ceftriaxone); Carbapenems (imipenem, meropenem, doripenem); MDR1, multidrugresistance (must test either intermediate $[\mathrm{I}]$ or resistant $[\mathrm{R}]$ to at least 1 drug in 3 of the 5 following classes [ESC4, FQ3, AMINO, carbapenems, \& PIP/PIPTAZ]); FQ3, fluoroquinolones (ciprofloxacin, levofloxacin, moxifloxacin); AMINOS, aminoglycosides (amikacin, gentamicin, tobramycin); ESC2, extended-spectrum cephalosporin (cefepime, ceftazidime); FQ2, fluoroquinolones (ciprofloxacin, levofloxacin); PIP, piperacillin; PIPTAZ, piperacillin/tazobactam; MDR2, multidrug-resistance (must test either I or R to at least 1 drug in 3 of the 5 following classes [ESC2, FQ2, AMINOS, carbapenems, \& PIP/PIPTAZ]); MDR3, multidrug-resistance (must test either I or R to at least 1 drug in 3 of the 6 following classes [ESC4, FQ2, AMINOS, carbapenems, PIP/PIPTAZ, \& ampicillin/sulbactam]).

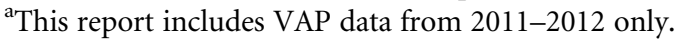

${ }^{\mathrm{b}}$ If the percent of isolates tested is less than $70 \%$, caution should be used when interpreting the percent resistance.

${ }^{\mathrm{c}}$ Percent resistance is calculated only when at least 20 isolates have been tested.

resistance report to include data representative of almost all acute care hospitals, LTACHs, and IRFs in the United States, a reporting milestone made possible by the increase in NHSN's surveillance coverage in 2011-2014 as a result of expanding federal and state HAI reporting requirements. The CMS Hospital Inpatient QRP mandated the reporting of CLABSIs among critical care patients starting in January 2011, and the reporting of CAUTIs in critical care patients and SSIs following abdominal hysterectomies and colon surgeries in January 2012. CMS mandated CLABSI and
CAUTI reporting from LTACHs and CAUTI reporting from IRFs in October 2012. Although this report may not be representative of the entire US patient population, CMS QRPs and numerous state mandates have helped to increase the consistency and applicability of the reported data, allowing this report to provide the first comprehensive national picture of antimicrobial resistance from clinically relevant infections reported to NHSN. The data in this report can be considered a national benchmark for HAI antimicrobial resistance among select phenotypes. 
TA B LE 9. Percent of Pathogens Reported From Surgical Site Infections (SSIs) That Tested Resistant to Selected Antimicrobial Agents, by Period, 2011-2014

\begin{tabular}{|c|c|c|c|c|c|c|c|c|c|c|c|c|}
\hline \multirow[b]{2}{*}{ Pathogen, antimicrobial } & \multicolumn{3}{|c|}{2011} & \multicolumn{3}{|c|}{2012} & \multicolumn{3}{|c|}{2013} & \multicolumn{3}{|c|}{2014} \\
\hline & $\begin{array}{l}\text { No. of isolates } \\
\text { reported }\end{array}$ & $\begin{array}{l}\% \text { of isolates } \\
\text { tested }^{\mathrm{a}}\end{array}$ & $\begin{array}{c}\% \\
\text { Resistance }\end{array}$ & $\begin{array}{l}\text { No. of isolates } \\
\text { reported }\end{array}$ & $\begin{array}{l}\% \text { of isolates } \\
\text { tested }^{\mathrm{a}}\end{array}$ & $\begin{array}{c}\% \\
\text { Resistance }\end{array}$ & $\begin{array}{l}\text { No. of isolates } \\
\text { reported }\end{array}$ & $\begin{array}{l}\% \text { of isolates } \\
\text { tested }^{\mathrm{a}}\end{array}$ & $\begin{array}{c}\% \\
\text { Resistance }\end{array}$ & $\begin{array}{l}\text { No. of isolates } \\
\text { reported }\end{array}$ & $\begin{array}{l}\% \text { of isolates } \\
\text { tested }^{\mathrm{a}}\end{array}$ & $\begin{array}{c}\% \\
\text { Resistance }\end{array}$ \\
\hline Staphylococcus aureus & 5,152 & & & 8,435 & & & 8,577 & & & 8,738 & & \\
\hline OX/METH/CEFOX & & 96.2 & 42.7 & & 94.8 & 44.7 & & 94.8 & 44.2 & & 94.3 & 42.6 \\
\hline Enterococcus spp. & & & & & & & & & & & & \\
\hline E. faecium & 414 & 973 & 640 & 1,123 & 063 & 597 & 1,261 & 962 & 606 & 1,342 & 050 & 58.4 \\
\hline E. faecalis & 1,192 & (31.0 & 04.0 & 2,936 & 90.5 & 39.1 & 3,474 & 9.2 & 00.0 & 3,554 & 95.9 & 58.4 \\
\hline VAN & & 94.0 & 5.3 & & 93.8 & 3.9 & & 93.8 & 3.7 & & 93.6 & 3.5 \\
\hline Klebsiella (pneumoniae/oxytoca) & 831 & & & 1,874 & & & 2,043 & & & 2,319 & & \\
\hline ESC4 & & 81.7 & 10.6 & & 78.8 & 9.7 & & 80.4 & 9.6 & & 81.1 & 11.3 \\
\hline Carbapenems & & 59.6 & 4.6 & & 66.9 & 3.0 & & 67.6 & 3.4 & & 66.0 & 3.3 \\
\hline MDR1 & & 86.8 & 5.8 & & 89.2 & 4.6 & & 92.4 & 4.4 & & 92.4 & 4.6 \\
\hline Escherichia coli & 1,940 & & & 5,307 & & & 6,366 & & & 6,816 & & \\
\hline ESC4 & & 76.6 & 13.3 & & 79.0 & 13.1 & & 81.2 & 14.0 & & 81.2 & 15.3 \\
\hline FQ3 & & 93.6 & 29.1 & & 94.1 & 29.6 & & 94.4 & 31.4 & & 94.0 & 30.9 \\
\hline Carbapenems & & 60.6 & 0.9 & & 66.9 & 0.9 & & 67.5 & 0.7 & & 66.8 & 0.7 \\
\hline MDR1 & & 85.4 & 6.1 & & 89.5 & 6.0 & & 91.7 & 6.7 & & 92.7 & 6.5 \\
\hline Enterobacter spp. & 866 & & & 1,769 & & & 1,924 & & & 2,056 & & \\
\hline ESC4 & & 92.4 & 27.9 & & 91.1 & 26.1 & & 92.9 & 28.0 & & 94.0 & 27.5 \\
\hline Carbapenems & & 61.5 & 2.6 & & 65.9 & 2.4 & & 68.2 & 4.0 & & 67.3 & 3.4 \\
\hline MDR1 & & 91.6 & 2.6 & & 93.4 & 2.5 & & 95.1 & 3.1 & & 95.4 & 2.4 \\
\hline Pseudomonas aeruginosa & 1,056 & & & 2,285 & & & 2,500 & & & 2,617 & & \\
\hline AMINOS & & 91.9 & 8.4 & & 96.3 & 8.0 & & 97.3 & 7.6 & & 96.3 & 6.6 \\
\hline ESC2 & & 92.6 & 11.7 & & 93.7 & 10.4 & & 94.8 & 10.4 & & 94.0 & 9.9 \\
\hline $\mathrm{FQ} 2$ & & 94.8 & 14.1 & & 94.5 & 13.0 & & 94.8 & 11.9 & & 94.8 & 11.5 \\
\hline Carbapenems & & 76.3 & 7.8 & & 78.6 & 9.5 & & 78.2 & 9.1 & & 76.5 & 7.7 \\
\hline PIP/ PIPTAZ & & 76.5 & 8.0 & & 77.0 & 8.2 & & 88.4 & 6.9 & & 89.8 & 7.4 \\
\hline MDR2 & & 95.5 & 5.3 & & 96.1 & 5.5 & & 96.9 & 4.3 & & 96.0 & 4.3 \\
\hline Acinetobacter spp. & 102 & & & 161 & & & 177 & & & 174 & & \\
\hline Carbapenems & & 70.6 & 45.8 & & 76.4 & 36.6 & & 73.4 & 33.1 & & 77.6 & 33.3 \\
\hline MDR3 & & 95.1 & 40.2 & & 95.0 & 39.2 & & 94.4 & 33.5 & & 97.7 & 32.9 \\
\hline
\end{tabular}

NOTE. OX/METH/CEFOX, oxacillin/methicillin/cefoxitin; VAN, vancomycin; ESC4, extended-spectrum cephalosporin (cefepime, cefotaxime, ceftazidime, ceftriaxone); Carbapenems (imipenem, meropenem, doripenem); MDR1, multidrug-resistance (must test either intermediate [I] or resistant [R] to at least 1 drug in 3 of the 5 following classes [ESC4, FQ3, AMINO, carbapenems, \& PIP/PIPTAZ]); FQ3, fluoroquinolones (ciprofloxacin, levofloxacin, moxifloxacin); AMINOS, aminoglycosides (amikacin, gentamicin, tobramycin); ESC2, extendedspectrum cephalosporin (cefepime, ceftazidime); FQ2, fluoroquinolones (ciprofloxacin, levofloxacin); PIP, piperacillin; PIPTAZ, piperacillin/tazobactam; MDR2, multidrug-resistance (must test either I or R to at least 1 drug in 3 of the 5 following classes [ESC2, FQ2, AMINOS, carbapenems, \& PIP/PIPTAZ]); MDR3, multidrug-resistance (must test either I or R to at least 1 drug in 3 of the 6 following classes [ESC4, FQ2, AMINOS, carbapenems, PIP/PIPTAZ, \& ampicillin/sulbactam]).

${ }^{\mathrm{a}}$ If the percent of isolates tested is less than $70 \%$, caution should be used when interpreting the percent resistance. 
TABLE 10. Effect of a New Carbapenem-Resistant Enterobacteriaceae (CRE) Definition on the Percent Resistance, by HealthcareAssociated Infection Type and Pathogen Reported to the National Healthcare Safety Network (NHSN), 2014

\begin{tabular}{|c|c|c|c|c|c|c|c|c|c|}
\hline \multirow[b]{2}{*}{$\begin{array}{l}\text { CRE pathogen, } \\
\text { CRE definition }\end{array}$} & \multicolumn{3}{|c|}{ CLABSI } & \multicolumn{3}{|c|}{ CAUTI } & \multicolumn{3}{|c|}{ SSI } \\
\hline & $\begin{array}{c}\text { No. of isolates } \\
\text { reported }\end{array}$ & $\begin{array}{c}\% \text { of isolates } \\
\text { tested }^{\mathrm{c}}\end{array}$ & $\begin{array}{c}\% \\
\text { Resistance }\end{array}$ & $\begin{array}{l}\text { No. of isolates } \\
\text { reported }\end{array}$ & $\begin{array}{c}\% \text { of isolates } \\
\text { tested }^{\mathrm{c}}\end{array}$ & $\begin{array}{c}\% \\
\text { Resistance }\end{array}$ & $\begin{array}{c}\text { No. of isolates } \\
\text { reported }\end{array}$ & $\begin{array}{c}\% \text { of isolates } \\
\text { tested }^{\mathrm{c}}\end{array}$ & $\begin{array}{c}\% \\
\text { Resistance }\end{array}$ \\
\hline Klebsiella (pneumoniae/oxytoca) & 2,200 & & & 4,725 & & & 2,319 & & \\
\hline Future $^{\mathrm{a}^{\mathrm{a}}}$ & & 77.0 & 10.0 & & 73.9 & 9.3 & & 72.6 & 2.9 \\
\hline Current $^{\mathrm{b}}$ & & 73.3 & 10.9 & & 68.8 & 9.5 & & 66.0 & 3.3 \\
\hline Escherichia coli & 1,595 & & & 10,840 & & & 6,816 & & \\
\hline Future $^{\mathrm{a}}$ & & 75.0 & 1.4 & & 71.5 & 0.6 & & 73.1 & 0.4 \\
\hline Current $^{\mathrm{b}}$ & & 70.9 & 1.9 & & 66.6 & 1.1 & & 66.8 & 0.7 \\
\hline Enterobacter spp. & 1,069 & & & 1,641 & & & 2,056 & & \\
\hline Future $^{\mathrm{a}}$ & & 76.0 & 5.2 & & 75.8 & 6.3 & & 74.5 & 2.0 \\
\hline Current $^{\mathrm{b}}$ & & 70.7 & 6.6 & & 70.7 & 6.5 & & 67.3 & 3.4 \\
\hline All CRE & 4,864 & & & 17,206 & & & 11,191 & & \\
\hline Future $^{\mathrm{a}}$ & & 76.1 & 6.2 & & 72.6 & 3.6 & & 73.3 & 1.2 \\
\hline Current $^{\mathrm{b}}$ & & 71.9 & 7.1 & & 67.6 & 4.0 & & 66.7 & 1.8 \\
\hline
\end{tabular}

NOTE. CAUTI, catheter-associated urinary tract infection; CLABSI, central line-associated bloodstream infection; SSI, surgical site infection. ${ }^{\mathrm{a}}$ In future iterations of this report, the Centers for Disease Control and Prevention will use an updated definition for carbapenem-resistant Enterobacteriaceae (CRE). The future CRE definition includes any Klebsiella pneumoniae, Klebsiella oxytoca, Escherichia coli, or Enterobacter spp. that tested resistant $[\mathrm{R}]$ to imipenem, meropenem, doripenem, or ertapenem.

${ }^{\mathrm{b}}$ Current definition of CRE includes any Klebsiella pneumoniae, Klebsiella oxytoca, Escherichia coli, or Enterobacter spp. that tested intermediate $[\mathrm{I}]$ or resistant $[\mathrm{R}]$ to imipenem, meropenem, or doripenem.

${ }^{c}$ If the percent of isolates tested is less than $70 \%$, caution should be used when interpreting the percent resistance.

Compared with earlier reports, an increasing proportion of data was reported from LTACHs, critical access hospitals, and IRFs. ${ }^{2}$ Although device-associated HAI surveillance has increased in ward locations in recent years, most pathogens were reported from critical care units. In January 2015, CMS expanded the reporting requirements for hospitals to include CLABSIs and CAUTIs from adult and pediatric medical, surgical, and medical/surgical wards. As reporting requirements expand to additional locations, analyses will become more inclusive of varying patient populations. In addition, as reporting increases from different facility types, analyses will allow for a more accurate assessment of how widespread any one resistant phenotype is among facility types, and how successful facilities and states have been in curtailing the spread of resistant phenotypes.

There have been some changes in the distribution of reported pathogens compared with previous reports. ${ }^{2}$ With the increase in reporting of CAUTIs due to CMS QRP requirements, E. coli became the most common HAI pathogen, and an increase in the reporting of yeast was seen. However, in January 2015, NHSN's definition of CAUTI was modified such that only those events in which bacteria are identified as causative organisms are considered CAUTIs. This change will eliminate Candida spp. and other yeast reported for CAUTIs in future years; however, these organisms will continue to be reported and tracked among the other NHSN infection types. The relative proportions of Acinetobacter spp. and Serratia spp. decreased and were no longer among the 15 most prevalent species reported across all HAIs. Both Bacteroides spp. and viridans streptococci emerged as prevalent SSI pathogens in 2011-2014, and were commonly reported from abdominal and neck procedures, respectively.
Although no statistical tests for significance were performed on the 4 years of data included in this report, there were clinically meaningful changes in the magnitude of the percent resistance worth noting that highlight areas to monitor closely in future years. The magnitude of the resistance percentages among Acinetobacter spp. appears to be decreasing in recent years across all HAI types. The cause of a decrease and whether or not it represents a true decrease in the prevalence of resistant pathogens are not known. Increases were seen in the proportion of E. coli isolated from device-associated HAIs that tested resistant to fluoroquinolones, extended-spectrum cephalosporins, and those identified as multidrug-resistant. This could be reflective of the spread of E. coli sequence type 131 (ST131), which often produces extended-spectrum B-lactamases and is frequently resistant to fluoroquinolones. ${ }^{15,16}$

Also of note is the declining percentage of Enterobacteriaceae isolates with reported susceptibility test results for carbapenems. This may be due to cascade testing practices within laboratories and/or implementation of various suppression rules in the display of carbapenem test results. Regardless, the magnitude of the resistance percentage for carbapenem-resistant Enterobacter spp. appears to have increased slightly in recent years, whereas carbapenem-resistant Klebsiella and E. coli have remained more level. CRE continues to be an important cause of HAIs, and these data highlight the need to respond aggressively to prevent further transmission. CDC's guidelines for CRE management, including a CRE Prevention Toolkit, can be found at http://www.cdc.gov/ HAI/organisms/cre/index.html.

The updated CRE definition revealed a slight decrease in the percent resistance compared with the current definition. This decrease was driven by an increase in the number of isolates included in the denominator (ie, number tested), because the 
updated CRE definition captured additional isolates with susceptibility data for only ertapenem. Although there were some increases in the number of isolates counted as CRE, this had a minimal effect on the resistance percentage owing to the removal of pathogens from the numerator that tested intermediate to carbapenems.

Our results are subject to limitations. As antimicrobial resistance data captured in NHSN are representative of almost all clinical laboratories in the country, differences may exist in the testing and reporting methods between laboratories that could cause inconsistencies in the reported data. NHSN captures only the category interpretation and not the measured minimum inhibitory concentration, and we therefore are unable to account for differences in the interpretations of breakpoints between laboratories. In addition, some facilities may perform selective testing of broad-spectrum agents or have suppression rules in place that prevent testing results from being readily available to NHSN data entry personnel. Although this may result in some selection bias, any inflation of proportions is likely to be small because most reported isolates had testing results for most phenotypes.

These data represent a current assessment of the prevalence of antimicrobial-resistant phenotypes associated with HAIs reported to NHSN across over 4,500 healthcare facilities in the United States. In a recent report, CDC estimated that $14 \%$ of all HAIs that occurred in acute care hospitals in 2014 were caused by an antibiotic-resistant threat pathogen. ${ }^{17}$ The data shown in this report, used in conjunction with other available data, should alert the infection prevention community to the need for vigilance in the identification and implementation of appropriate infection control and antimicrobial stewardship activities as they address the challenges caused by antimicrobial resistance in their facilities, jurisdictions, regions, and across the nation.

\section{ACKNOWLEDGMENTS}

We thank the NHSN participants and the infection control community for their ongoing efforts to monitor infections and improve patient safety, and our colleagues in the Division of Healthcare Quality Promotion, who work to support this unique and growing public health network.

Financial support. The NHSN surveillance system is supported by the Division of Healthcare Quality Promotion, CDC.

Potential conflicts of interest. All authors report no conflicts of interest relevant to this article.

Disclaimer: The findings and conclusions in this report are those of the authors and do not necessarily represent the official position of the CDC or the Agency for Toxic Substances and Diseases Registry.

Address correspondence to Lindsey Weiner, MPH, 1600 Clifton Rd, MS A-24, Atlanta, GA 30329 (Lweiner@cdc.gov).

\section{REFERENCES}

1. Hidron AI, Edwards JR, Patel J, et al. Antimicrobial-resistant pathogens associated with healthcare-associated infections: annual summary of data reported to the National Healthcare Safety Network at the Centers for Disease Control and
Prevention, 2006-2007 (published correction appears in Infect Control Hosp Epidemiol 2009;30:107). Infect Control Hosp Epidemiol 2008;29:996-1011.

2. Sievert DM, Ricks P, Edwards JR, et al. Antimicrobial-resistant pathogens associated with healthcare-associated infections: summary of data reported to the National Healthcare Safety Network at the Centers for Disease Control and Prevention, 2009-2010. Infect Control Hosp Epidemiol 2013;34:1-14.

3. Dudeck MA, Edwards JR, Allen-Bridson K, et al. National Healthcare Safety Network report, data summary for 2013, Device-Associated Module. Am J Infect Control 2015;43:206-221.

4. Centers for Disease Control and Prevention (CDC). 2014 National and state healthcare-associated infections progress report. CDC website. http://www.cdc.gov/hai/progress-report/index.html. Published March 3, 2016. Accessed March 15, 2016.

5. Centers for Disease Control and Prevention (CDC). Antibiotic resistance threats in the United States, 2013. CDC website. http:// www.cdc.gov/drugresistance/threat-report-2013/pdf/ar-threats2013-508.pdf. Published September 2013. Accessed June 4, 2015.

6. Centers for Disease Control and Prevention (CDC). Bloodstream infection event (central line-associated bloodstream infection and non-central line-associated bloodstream infection). CDC website. http://www.cdc.gov/nhsn/PDFs/pscManual/4PSC_CLABScurrent. pdf. Updated January 2016. Accessed March 15, 2016.

7. Centers for Disease Control and Prevention (CDC). Urinary tract infection (catheter-associated urinary tract infection [CAUTI] and non-catheter-associated urinary tract infection [UTI]) and other urinary system infection [USI]) events. CDC website. http://www.cdc.gov/nhsn/PDFs/pscManual/7pscCAUTIcurrent. pdf. Updated January 2016. Accessed March 15, 2016.

8. Centers for Disease Control and Prevention (CDC). Pneumonia (ventilator-associated [VAP] and non-ventilator-associated pneumonia [PNEU]) event. CDC website. http://www.cdc.gov/ nhsn/PDFs/pscManual/6pscVAPcurrent.pdf. Updated January 2016. Accessed March 15, 2016.

9. Centers for Disease Control and Prevention (CDC). Surgical site infection (SSI) event. CDC website. http://www.cdc.gov/nhsn/ PDFs/pscManual/9pscSSIcurrent.pdf. Updated January 2016. Accessed March 15, 2016.

10. Clinical and Laboratory Standards Institute (CLSI). Performance standards for antimicrobial susceptibility testing: 24th informational supplement. Wayne, PA: CLSI; 2014:M100-S24.

11. Magiorakos A-P, Srinivasan A, Carey RB, et al. Multidrug-resistant, extensively drug-resistant and pandrug-resistant bacteria: an international expert proposal for interim standard definitions for acquired resistance. Clin Microbiol Infect 2012;18:268-281.

12. Chea N, Bulens $\mathrm{SN}$, Kongphet-Tran $\mathrm{T}$, et al. Improved phenotype-based definition for identifying carbapenemase producers among carbapenem-resistant Enterobacteriaceae. Emerg Infect Dis 2015;21:1611-1616.

13. Centers for Disease Control and Prevention (CDC). NHSN e-News. http://www.cdc.gov/nhsn/PDFs/Newsletters/vol9-3-eNL-Sept-2014. pdf. CDC website. Updated September 2014. Accessed August 31, 2015.

14. Council of State and Territorial Epidemiologists (CSTE). Standardized definition for carbapenem-resistant Enterobacteriaceae (CRE) and recommendation for sub-classification and stratified reporting. http://c.ymcdn.com/sites/www.cste.org/resource/resmgr/2015PS/ 2015PSFinal/15-ID-05.pdf. Accessed September 2, 2015. 
15. Nicolas-Chanoine $\mathrm{MH}$, Blanco J, Leflon-Guibout V, et al. Intercontinental emergence of Escherichia coli clone O25:H4-ST131 producing CTX-M-15. J Antimicrob Chemother 2008;61:273-281.

16. Johnson JR, Nicolas-Chanoine MH, DebRoy C, et al. Comparison of Escherichia coli ST131 pulsotypes, by epidemiologic traits,
1967-2009. Emerg Infect Dis 2012, http://dx.doi.org/10.3201/ eid1804.111627. Accessed March 11, 2016.

17. Weiner LM, Fridkin SK, Aponte-Torres Z, et al. Vital signs: preventing antibiotic-resistant infections in hospitals-United States, 2014. MMWR Morb Mortal Wkly Rep 2016;65:235-241. 